TRANSACTIONS OF THE

AMERICAN MATHEMATICAL SOCIETY

Volume 357 , Number 10, Pages 4159-4174

S 0002-9947(05)03905-X

Article electronically published on May 20, 2005

\title{
PRESCRIBING ANALYTIC SINGULARITIES FOR SOLUTIONS OF A CLASS OF VECTOR FIELDS ON THE TORUS
}

\author{
ADALBERTO P. BERGAMASCO AND SÉRGIO LUÍS ZANI
}

\begin{abstract}
We consider the operator $L=\partial_{t}+(a(t)+i b(t)) \partial_{x}$ acting on distributions on the two-torus $\mathbb{T}^{2}$, where $a$ and $b$ are real-valued, real analytic functions defined on the unit circle $\mathbb{T}^{1}$. We prove, among other things, that when $b$ changes sign, given any subset $\Sigma$ of the set of the local extrema of the local primitives of $b$, there exists a singular solution of $L$ such that the $t$-projection of its analytic singular support is $\Sigma$; furthermore, for any $\tau \in \Sigma$ and any closed subset $F$ of $\mathbb{T}_{x}^{1}$ there exists $u \in \mathcal{D}^{\prime}\left(\mathbb{T}^{2}\right)$ such that $L u \in C^{\omega}\left(\mathbb{T}^{2}\right)$ and sing $\operatorname{supp}_{\mathrm{A}}(u)=\{\tau\} \times F$. We also provide a microlocal result concerning the trace of $u$ at $t=\tau$.
\end{abstract}

\section{INTRODUCTION}

There are many works about hypoellipticity, local or global, smooth or analytic. In the global case we cite [B], [BCM], BNZ1], [BNZ2], DGY], G], GW], $\mathrm{Bi}],[\mathrm{Ho}$, and $[\mathrm{M}]$. In these papers, in order to prove that some of the operators under study are not hypoelliptic, singular solutions are constructed. By a singular solution of an operator $L$, we mean a nonanalytic (nonsmooth) distribution $u$ such that $L u$ is analytic (smooth). However, not much is known about the location and nature of the singularities. The main goal of the present work is to help fill this gap for a class of vector fields on the two-torus in the global analytic setting as we describe below.

Let $c=a+i b$, where $a$ and $b$ are real-valued, real analytic functions defined on the unit circle $\mathbb{T}^{1}$. We consider the operator

$$
L=\partial_{t}+c(t) \partial_{x}
$$

acting on distributions on the two-torus $\mathbb{T}^{2}$.

We recall that $L$ is said to be globally analytic hypoelliptic $(\mathrm{GAH})$ if one has $u \in C^{\omega}\left(\mathbb{T}^{2}\right)$ whenever $u \in \mathcal{D}^{\prime}\left(\mathbb{T}^{2}\right)$ and $L u \in C^{\omega}\left(\mathbb{T}^{2}\right)$.

In $\mathrm{B}$. one finds a characterization of the global analytic hypoellipticity of the operator $L$; in particular, it is proved there that if $b \not \equiv 0$, then $L$ is globally analytic hypoelliptic if and only if the function $b$ does not change sign (the Nirenberg-Treves condition $(\mathcal{P})$; see $[\mathrm{T} 2]$ ), or equivalently, no primitive of $b$ defined in $\mathbb{R}$ has local extrema.

Received by the editors December 9, 2003.

2000 Mathematics Subject Classification. Primary 35A20, 35H10.

Key words and phrases. Analytic singularities, global analytic hypoellipticity, stationary phase.

The first author was partially supported by CNPq. Both authors were partially supported by FAPESP.

(C)2005 American Mathematical Society Reverts to public domain 28 years from publication 
We emphasize that the method applied in $[\mathrm{B}]$ does not yield all the possible singularities. We now mention two examples of this difficulty. Assume that $b \not \equiv 0$; let $B$ be one of its primitives in $\mathbb{R}$ and suppose that the origin is a local minimum of $B$. If $t_{1}, t_{2} \in(0,2 \pi)$ are points of local maximum of $B_{\mid[0,2 \pi]}$, with $B\left(t_{1}\right)<$ $B\left(t_{2}\right)$, then the singular solution constructed in $\left[\mathrm{B}\right.$ is real analytic when $t=t_{1}$. Similarly, if $t_{1}, t_{2} \in(0,2 \pi)$ are points of global maximum of $B_{\mid[0,2 \pi]}$, then the solution constructed in $[\overrightarrow{\mathrm{B}}]$ is singular at both points $\left(t_{1}, 0\right)$ and $\left(t_{2}, 0\right)$.

In fact, if $b \not \equiv 0$ and $t_{\star}$ is a point of local maximum of $B$, then in Lemma 2.1 we construct $u \in \mathcal{D}^{\prime}\left(\mathbb{T}^{2}\right)$ with sing $\operatorname{supp}_{\mathrm{A}}(u)=\left\{\left(t_{\star}, 0\right)\right\}$ and $L u \in C^{\omega}\left(\mathbb{T}^{2}\right)$ and, furthermore, $u\left(t_{\star}, x\right)=\delta_{+}(x) \doteq \sum_{n \geq 1} e^{i n x}$. More generally (see Theorem 2.2), given, instead of $\delta_{+}$, a distribution $\widetilde{v}(x)=\sum_{n>1} v_{n} e^{i n x}$, there is $u \in \mathcal{D}^{\prime}\left(\mathbb{T}^{2}\right)$ such that its analytic singular support is $\operatorname{sing} \operatorname{supp}_{\mathrm{A}}(u)=\left\{t_{\star}\right\} \times \operatorname{sing} \operatorname{supp}_{\mathrm{A}}(\widetilde{v})$ and $L u \in$ $C^{\omega}\left(\mathbb{T}^{2}\right)$. Moreover (see Theorem [2.3), given any nonempty closed subset $F \subset \mathbb{T}^{1}$, we construct $u \in \mathcal{D}^{\prime}\left(\mathbb{T}^{2}\right)$ such that $L u \in C^{\omega}\left(\mathbb{T}^{2}\right)$ and $\operatorname{sing} \operatorname{supp}_{\mathrm{A}}(u)=\left\{t_{\star}\right\} \times F$; in fact (see Remark 2.4), the analytic wave front set of the trace $u\left(t_{\star}, \cdot\right)$ is equal to $F \times \mathbb{R}_{+}$.

We also have the analogous results for the local minima. For instance, if $s_{\star}$ is such a point and $F \subset \mathbb{T}^{1}$ is a nonempty closed set, then there is $u \in \mathcal{D}^{\prime}\left(\mathbb{T}^{2}\right)$ with $\operatorname{sing} \operatorname{supp}_{\mathrm{A}}(u)=\left\{s_{\star}\right\} \times F$ and $L u \in C^{\omega}\left(\mathbb{T}^{2}\right)$ and, furthermore, the analytic wave front set of the trace $u\left(s_{\star}, \cdot\right)$ is equal to $F \times \mathbb{R}_{-}$.

Theorems 2.9 and 2.10 put together the above results in a more complete way. As an example, suppose $b \not \equiv 0$ and let $B: \mathbb{R} \rightarrow \mathbb{R}$ be a real analytic function such that $B^{\prime}=b$. Let $\Sigma$ be a nonempty subset of the set of points in $[0,2 \pi)$ which are local extrema of $B$. Then there exists a singular solution $u$ such that sing $\operatorname{supp}_{A}(u)=\Sigma \times\{0\}$.

Our results are sharp. Indeed, it follows from [T1 and also from [BT] that if $t$ is neither a local maximum nor a local minimum, then every $u \in \mathcal{D}^{\prime}\left(\mathbb{T}^{2}\right)$ such that $L u \in C^{\omega}\left(\mathbb{T}^{2}\right)$ is real analytic at $(t, x)$ for arbitrary $x \in \mathbb{T}^{1}$. Furthermore, concerning the microlocal aspect of the singularities, the results of [BT] imply that, for instance, in the case of a local maximum $t_{\star}$, the trace at $t=t_{\star}$ of any singular solution has an analytic wave-front set contained in $\mathbb{T}^{1} \times \mathbb{R}_{+}$.

We stress that besides constructing solutions with prescribed singularities, the present work yields a new, simpler, proof of the the hardest case of the main result about global analytic hypoellipticity in $[\overline{\mathrm{B}}$, that is, when $b \not \equiv 0$. In both works the proofs rely on the method of stationary phase, but now we make use of a suitable function $\psi$ (see the proof of Lemma 2.1) whose role is to simplify the application of this method, similar to what was done in [BNZ1].

We point out that our interest lies primarily in the nonreal case; for the sake of completeness, we also provide, in section 3 , information about singularities of solutions when $b \equiv 0$.

For later use, we define $c_{0}=\frac{1}{2 \pi} \int_{0}^{2 \pi} c(t) d t, a_{0}=\Re c_{0}$, and $b_{0}=\Im c_{0}$.

\section{Construction of singular solutions in the Case $b \not \equiv 0$}

Suppose that $L=\partial_{t}+(a(t)+i b(t)) \partial_{x}$ is not GAH and that $b \not \equiv 0$; equivalently, suppose that $b$ changes sign. Let $B: \mathbb{R} \rightarrow \mathbb{R}$ be a primitive of $b$. Then the function has at least one point of local maximum. In fact, there exist $r \in \mathbb{Z}_{+}$and points $t_{0}, \ldots, t_{r}$, with $t_{0}<t_{1}<\cdots<t_{r}$, and $t_{r}-t_{0}<2 \pi$, such that any point of local maximum of $B$ is equal to $t_{k}+2 j \pi$, for some $k=0, \ldots, r$ and some $j \in \mathbb{Z}$. 
Similarly we have points of local minimum $s_{0}, \ldots, s_{r}$ such that, by setting $t_{r+1} \doteq$ $t_{0}+2 \pi$ and $s_{r+1} \doteq s_{0}+2 \pi$, we may write

$$
s_{0}<t_{0}<s_{1}<t_{1}<s_{2}<\cdots<s_{r}<t_{r}<s_{r+1}<t_{r+1} .
$$

We will use the same notation, namely, $t_{j}$ and $s_{j}$, for the corresponding zeros of $b$ in $\mathbb{T}^{1}$.

The following crucial lemma will be proved later in this section.

Lemma 2.1. Suppose that $L$ is not $G A H$ and that $b \not \equiv 0$. Let $t_{\star} \in\left\{t_{0}, \ldots, t_{r}\right\}$. Then there exists $u \in \mathcal{D}^{\prime}\left(\mathbb{T}^{2}\right)$ with $\operatorname{sing} \operatorname{supp}_{\mathrm{A}}(u)=\left\{\left(t_{\star}, 0\right)\right\}$ and $L u \in C^{\omega}\left(\mathbb{T}^{2}\right)$. More precisely, $u$ can be chosen so that $u\left(t_{\star}, x\right)=\delta_{+}(x) \doteq \sum_{n \geq 1} e^{i n x}$.

Assuming the previous lemma we can prove

Theorem 2.2. Suppose that $L$ is not $G A H$ and $b \not \equiv 0$. Let $t_{\star} \in\left\{t_{0}, \ldots, t_{r}\right\}$. Given a sequence $\left(v_{n}\right)_{n \in \mathbb{Z}}$ such that $v_{n}$ is tempered in $n \in \mathbb{Z}$, but not exponentially decaying as $n \rightarrow+\infty$, although exponentially decaying in the opposite direction, then there exists $v \in \mathcal{D}^{\prime}\left(\mathbb{T}^{2}\right) \backslash C^{\omega}\left(\mathbb{T}^{2}\right)$ such that $\widehat{v}\left(t_{\star}, n\right)=v_{n}, L v \in C^{\omega}\left(\mathbb{T}^{2}\right)$, and $\operatorname{sing} \operatorname{supp}_{\mathrm{A}}(v)=\left\{t_{\star}\right\} \times \operatorname{sing} \operatorname{supp}_{\mathrm{A}}(\widetilde{v})$, where $\widetilde{v}(x) \doteq \sum_{n \in \mathbb{Z}} v_{n} e^{i n x}$.

Proof. It suffices to take $u$ as in Lemma 2.1] and define

$$
v(t, x)=\sum_{n \leq 0} v_{n} e^{i n x}+\sum_{n \geq 1} \widehat{u}(t, n) v_{n} e^{i n x} .
$$

In Lemma 4.1 we prove that for any given nonempty closed subset $F$ of $\mathbb{T}^{1}$, there exists $v_{o} \in \mathcal{D}^{\prime}\left(\mathbb{T}^{1}\right)$ such that sing $\operatorname{supp}_{\mathrm{A}}\left(v_{o}\right)=F$ and $v_{o}(x)=\sum_{n \geq 1} \widehat{v_{o}}(n) e^{i n x}$. We obtain

Theorem 2.3. Suppose that $L$ is not $G A H$ and $b \not \equiv 0$. Let $t_{\star} \in\left\{t_{0}, \ldots, t_{r}\right\}$ and let $F$ be a nonempty closed subset of $\mathbb{T}^{1}$. Then there exists $v \in \mathcal{D}^{\prime}\left(\mathbb{T}^{2}\right) \backslash C^{\omega}\left(\mathbb{T}^{2}\right)$ such that $L v \in C^{\omega}\left(\mathbb{T}^{2}\right)$ and $\operatorname{sing} \operatorname{supp}_{\mathrm{A}}(v)=\left\{t_{\star}\right\} \times F$. Furthermore, $\widehat{v}\left(t_{\star}, n\right)$ is tempered in $n \in \mathbb{Z}$ and decays exponentially as $n \rightarrow-\infty$.

Proof. Take $v_{o} \in \mathcal{D}^{\prime}\left(\mathbb{T}^{1}\right)$ as in Lemma 4.1 corresponding to the given closed subset $F$. Define $v_{n}={\widehat{v_{o}}}_{(}(n)$ for $n \geq 1$ and $v_{n}=0$ otherwise. Applying Theorem 2.2 the result follows immediately.

Remark 2.4. Note that the singularity of $v$ in Theorem 2.2 and Theorem 2.3 is due to the positive frequencies $n$. Moreover, any singular solution $w$ of $L$ such that $t_{\star}$ is in the $t$-projection of its analytic singular support has $\widehat{w}\left(t_{\star}, n\right)$ decaying exponentially as $n \rightarrow-\infty$; indeed, this follows from the analogue of Lemma 2.11 with negative indices. Thus, the analytic wave front set of the trace $w\left(t_{\star}, \cdot\right)$ is equal to $F \times \mathbb{R}_{+}$.

We now proceed to state, without proof, the analogous results for the case of local minima.

Lemma 2.5. Suppose that $L$ is not $G A H$ and that $b \not \equiv 0$. Let $s_{\star} \in\left\{s_{0}, \ldots, s_{r}\right\}$. Then there exists $u \in \mathcal{D}^{\prime}\left(\mathbb{T}^{2}\right)$ satisfying sing $\operatorname{supp}_{\mathrm{A}}(u)=\left\{\left(s_{\star}, 0\right)\right\}$ and $L u \in C^{\omega}\left(\mathbb{T}^{2}\right)$. More precisely, $u$ can be chosen so that $u\left(s_{\star}, x\right)=\delta_{-}(x) \doteq \sum_{n \leq-1} e^{\text {inx }}$. 
Theorem 2.6. Suppose that $L$ is not $G A H$ and $b \not \equiv 0$. Let $s_{\star} \in\left\{s_{0}, \ldots, s_{r}\right\}$. Given a sequence $\left(w_{n}\right)_{n \in \mathbb{Z}}$ such that $w_{n}$ is tempered, but not exponentially decaying as $n \rightarrow-\infty$, although exponentially decaying in the opposite direction, then there exists $w \in \mathcal{D}^{\prime}\left(\mathbb{T}^{2}\right) \backslash C^{\omega}\left(\mathbb{T}^{2}\right)$ such that $\widehat{w}\left(s_{\star}, n\right)=w_{n}, L w \in C^{\omega}\left(\mathbb{T}^{2}\right)$, sing $\operatorname{supp}_{\mathrm{A}}(w)=$ $\left\{s_{\star}\right\} \times \operatorname{sing} \operatorname{supp}_{\mathrm{A}}(\widetilde{w})$, where $\widetilde{w}(x) \doteq \sum_{n \in \mathbb{Z}} w_{n} e^{i n x}$.

Theorem 2.7. Suppose that $L$ is not $G A H$ and $b \not \equiv 0$. Let $s_{\star} \in\left\{s_{0}, \ldots, s_{r}\right\}$ and let $F$ be a nonempty closed subset of $\mathbb{T}^{1}$. Then there exists $w \in \mathcal{D}^{\prime}\left(\mathbb{T}^{2}\right) \backslash C^{\omega}\left(\mathbb{T}^{2}\right)$ such that $L w \in C^{\omega}\left(\mathbb{T}^{2}\right)$ and sing $\operatorname{supp}_{\mathrm{A}}(w)=\left\{s_{\star}\right\} \times F$. Furthermore, $\widehat{w}\left(s_{\star}, n\right)$ is tempered in $n \in \mathbb{Z}$ and decays exponentially as $n \rightarrow+\infty$.

Before proceeding to our main results (Theorems 2.9 and 2.10) we need the following

Lemma 2.8. For each $n \in \mathbb{Z}$ let $A_{n}=\left(a_{i j}(n)\right)$ be a $k \times k$ matrix such that $a_{i i}(n)=1$ and, for $i \neq j, a_{i j}(n)$ is exponentially decaying as $n \rightarrow \pm \infty$.

If $B_{n}$ is a $k \times 1$ matrix whose entries are tempered in $|n|$, then the equation $A_{n} X_{n}=B_{n}$ has, for all sufficiently large $|n|$, a unique solution. Furthermore, the entries of $X_{n}$ are tempered in $|n|$.

Proof. All we need is to observe that $\operatorname{det} A_{n}=1+\delta_{n}$ with $\delta_{n}$ decaying exponentially as $n \rightarrow \pm \infty$.

Theorem 2.9. Suppose that $L$ is not $G A H$ and $b \not \equiv 0$. Let $u_{j}(n), j=0, \ldots, 2 r+1$, be tempered in $n \in \mathbb{Z}$ and such that for $j=0, \ldots, r, u_{j}(n)$ decays exponentially as $n \rightarrow-\infty$, and for $j=r+1, \ldots, 2 r+1, u_{j}(n)$ decays exponentially as $n \rightarrow+\infty$.

Then there exists $u \in \mathcal{D}^{\prime}\left(\mathbb{T}^{2}\right)$ such that $L u \in C^{\omega}\left(\mathbb{T}^{2}\right)$ and $\widehat{u}\left(t_{j}, n\right)=u_{j}(n)$ for $j=0, \ldots, r$, and $\widehat{u}\left(s_{j-r}, n\right)=u_{j}(n)$, for $j=r+1, \ldots, 2 r+1$, for all $n$. Moreover,

$$
\subset\left(\bigcup_{j=0}^{r}\left\{t_{j}\right\} \times \operatorname{sing} \operatorname{supp}_{\mathrm{A}}\left(\widetilde{u}_{j}\right)\right) \cup\left(\bigcup_{j=1}^{r+1}\left\{s_{j}\right\} \times \operatorname{sing} \operatorname{supp}_{\mathrm{A}}\left(\widetilde{u}_{j}\right)\right),
$$

where $\widetilde{u}_{j}(x)=\sum_{n \in \mathbb{Z}} u_{j}(n) e^{i n x}, j=0, \ldots, 2 r+1$.

In particular, if $I$ is the set of indices $j \in\{0, \ldots, r\}$ such that $u_{j}(n)$ does not decay exponentially as $n \rightarrow+\infty$ and $J$ is the set of indices $j \in\{r+1, \ldots, 2 r\}$ such that $u_{j}(n)$ does not decay exponentially as $n \rightarrow-\infty$, then

$$
=\left(\bigcup_{j \in I}\left\{t_{j}\right\} \times \operatorname{sing} \operatorname{supp}_{\mathrm{A}}\left(\widetilde{u}_{j}\right)\right) \cup\left(\bigcup_{j \in J}\left\{s_{j}\right\} \times \operatorname{sing} \operatorname{supp}_{\mathrm{A}}\left(\widetilde{u}_{j}\right)\right) .
$$

Proof. For $j=0, \ldots, r$, we use Lemma 2.1 and obtain $v_{j} \in \mathcal{D}^{\prime}\left(\mathbb{T}^{2}\right)$ such that $L v_{j} \in C^{\omega}\left(\mathbb{T}^{2}\right), \widehat{v_{j}}\left(t_{j}, n\right)=1$, for all $n \geq 1, \widehat{v_{j}}\left(t_{j}, n\right)$ is exponentially decaying when $n \rightarrow-\infty$, and, for $t \neq t_{j}, \widehat{v}_{j}(t, n)$ is exponentially decaying as $|n| \rightarrow+\infty$.

For $j=r+1, \ldots, 2 r+1$, we use Lemma 2.5 and obtain $w_{j} \in \mathcal{D}^{\prime}\left(\mathbb{T}^{2}\right)$ such that $L w_{j} \in C^{\omega}\left(\mathbb{T}^{2}\right), \widehat{w_{j}}\left(s_{j-r}, n\right)=1$, for all $n \leq-1, \widehat{w}_{j}\left(s_{j-r}, n\right)$ is exponentially decaying when $n \rightarrow+\infty$, and, for $t \neq s_{j-r}, \widehat{w_{j}}(t, n)$ is exponentially decaying as $|n| \rightarrow+\infty$. 
Let $A_{n}=\left(a_{i j}(n)\right)$ be given by

$$
a_{i j}(n)=\left\{\begin{array}{l}
\widehat{v}_{i}\left(t_{j}, n\right), i=0, \ldots, r, \quad j=0, \ldots, r \\
\widehat{v}_{i}\left(s_{j-r}, n\right), i=0, \ldots, r, \quad j=r+1, \ldots, 2 r+1, \\
\widehat{w_{i}}\left(t_{j}, n\right), i=r+1, \ldots, 2 r+1, \quad j=0, \ldots, r \\
\widehat{w}_{i}\left(s_{j-r}, n\right), i=r+1, \ldots, 2 r+1, \quad j=r+1, \ldots, 2 r+1 .
\end{array}\right.
$$

Define $B_{n}=\left(b_{i 1}(n)\right)$, where $b_{i 1}(n)=u_{i}(n)$. Let $X_{n}=\left(\alpha_{i 1}(n)\right)$ be the solution of $A_{n} X_{n}=B_{n}$, for all $|n|>n_{0}$, for some $n_{0}$ as in Lemma 2.8 Then $v \in \mathcal{D}^{\prime}\left(\mathbb{T}^{2}\right)$ defined by

$$
\widehat{v}(t, n)=\sum_{i=0}^{r} \alpha_{i 1}(n) \widehat{v}_{i}(t, n)+\sum_{i=r+1}^{2 r+1} \alpha_{i 1}(n) \widehat{w_{i}}(t, n)
$$

satisfies $L v \in C^{\omega}\left(\mathbb{T}^{2}\right), \widehat{v}\left(t_{j}, n\right)=u_{j}(n)$ for $j=0, \ldots, r$, and $\widehat{v}\left(s_{j-r}, n\right)=u_{j}(n)$, for $j=r+1, \ldots, 2 r+1$, for $|n|>n_{0}$.

Let

$$
z_{j}= \begin{cases}e^{i t_{j}}, & j=0, \ldots, r, \\ e^{i s_{j-r}}, & j=r+1, \ldots, 2 r+1,\end{cases}
$$

and, for $|n| \leq n_{0}$, let $p_{n}(z)$ be a polynomial such that $p_{n}\left(z_{j}\right)=u_{j}(n), j=0, \ldots$, $2 r+1$.

Let $u(t, x)=v(t, x)+\sum_{|n| \leq n_{0}} p_{n}\left(e^{i t}\right) e^{i n x}$. Then $u \in \mathcal{D}^{\prime}\left(\mathbb{T}^{2}\right), L u \in C^{\omega}\left(\mathbb{T}^{2}\right)$ and $\widehat{u}\left(t_{j}, n\right)=u_{j}(n)$ for $j=0, \ldots, r$, and $\widehat{u}\left(s_{j-r}, n\right)=u_{j}(n)$, for $j=r+1, \ldots, 2 r+1$, for all $n$.

We also have

Theorem 2.10. Suppose that $L$ is not $G A H$ and $b \not \equiv 0$. Then, given $\left\{p_{1}, \ldots, p_{k}\right\} \subset$ $\left\{t_{0}, \ldots, t_{r}, s_{1}, \ldots, s_{r}\right\}$ and nonempty closed subsets $F_{j} \subset \mathbb{T}^{1}, j=1, \ldots, k$, there exists $u \in \mathcal{D}^{\prime}\left(\mathbb{T}^{2}\right)$ such that $L u \in C^{\omega}\left(\mathbb{T}^{2}\right)$ and $\operatorname{sing} \operatorname{supp}_{\mathrm{A}}(u)=\bigcup_{j=1}^{k}\left\{p_{j}\right\} \times F_{j}$.

Before we present the proof of Lemma 2.1, we need one more result.

Lemma 2.11. Let $c=a+i b$ be a continuous function defined on a bounded interval $J$, where $a$ and $b$ are real valued and $b$ has only isolated zeros. Let $C=A+i B$, where $A$ and $B$ are primitives of $a$ and $b$, respectively. Let $\left(f_{n}\right)_{n \in \mathbb{N}}$ be a sequence of continuous functions defined on $J$. Let $t_{0} \in J$ and set

$$
t_{1}=\inf \left\{t \in J ; B \text { is nondecreasing on }\left[t, t_{0}\right]\right\}
$$

and

$$
t_{2}=\sup \left\{t \in J ; B \text { is nonincreasing on }\left[t_{0}, t\right]\right\} .
$$

Then all the solutions of the $O D E$

$$
\frac{d}{d t} v_{n}(t)+i n c(t) v_{n}(t)=f_{n}(t)
$$

satisfy the following:

(1) If $v_{n}\left(t_{0}\right)$ is tempered in $n$ and if $f_{n}(t)$ is tempered in $n$ uniformly in $t$, then $v_{n}(t)$ is tempered in $n$ uniformly in $\left[t_{1}, t_{2}\right] \cap J$.

(2) If $v_{n}\left(t_{0}\right)$ is exponentially decaying in $n$ and if $f_{n}(t)$ is exponentially decaying in $n$ uniformly in $t$, then $v_{n}(t)$ is exponentially decaying in $n$ uniformly in $\left[t_{1}, t_{2}\right] \cap J$. 
(3) Suppose $t_{0}$ is not a minimum. If $v_{n}\left(t_{0}\right)$ is tempered in $n$ and if $f_{n}(t)$ is exponentially decaying in $n$ uniformly in $t$, then, for any sufficiently small $\varepsilon>0, v_{n}(t)$ is exponentially decaying in $n$ uniformly in

$$
\left[t_{1}, t_{2}\right] \cap J \backslash\left(t_{0}-\varepsilon, t_{0}+\varepsilon\right) .
$$

Proof. If $v_{n}$ is a solution of (2.1), then

$$
v_{n}(t)=e^{-i n\left(C(t)-C\left(t_{0}\right)\right)} v_{n}\left(t_{0}\right)+\int_{t_{0}}^{t} e^{-i n(C(t)-C(s))} f_{n}(s) d s, \quad t \in J .
$$

Note that for $t \in\left[t_{1}, t_{2}\right] \cap J$ we have $B(t) \leq B(s)$ whenever $s \in\left[t, t_{0}\right] \cap J$ or $s \in\left[t_{0}, t\right] \cap J$.

We prove statements (1) and (2). We have

$$
\left|v_{n}(t)\right| \leq\left|v_{n}\left(t_{0}\right)\right|+\sup _{s \in J}\left|f_{n}(s)\right| \ell(J),
$$

for all $t \in\left[t_{1}, t_{2}\right] \cap J, n \in \mathbb{N}$, where $\ell(J)$ is the length of $J$. It follows that $v_{n}(t)$ is tempered in $n$ for $t \in\left[t_{1}, t_{2}\right] \cap J$ in case (1) and exponentially decaying in $n$ for $t \in\left[t_{1}, t_{2}\right] \cap J$ in case $(2)$.

We now prove (3). Since $b$ has only isolated zeros, $B$ is strictly increasing in $\left[t_{1}, t_{0}\right] \cap J$ and strictly decreasing in $\left[t_{0}, t_{2}\right] \cap J$ (provided the intervals under consideration are nonempty).

Let $\varepsilon_{0}>0$ such that $J \backslash\left(t_{0}-\varepsilon_{0}, t_{0}+\varepsilon_{0}\right) \neq \emptyset$. Let $0<\varepsilon<\varepsilon_{0}$; if $t_{0}-\varepsilon \in J$ we set $\eta_{1}=B\left(t_{0}\right)-B\left(t_{0}-\varepsilon\right)>0$. If $t \in J \cap\left[t_{1}, t_{0}-\varepsilon\right]$, and $n \in \mathbb{N}$, we have

$$
\begin{aligned}
\left|v_{n}(t)\right| & \leq e^{n\left(B(t)-B\left(t_{0}\right)\right)}\left|v_{n}\left(t_{0}\right)\right|+\sup _{s \in J}\left|f_{n}(s)\right| \ell(J) \\
& \leq e^{-n \eta_{1}}\left|v_{n}\left(t_{0}\right)\right|+\sup _{s \in J}\left|f_{n}(s)\right| \ell(J) .
\end{aligned}
$$

Similarly, if $t \in J \cap\left[t_{0}+\varepsilon, t_{2}\right]$, we have $\eta_{2} \doteq B\left(t_{0}\right)-B\left(t_{0}+\varepsilon\right)>0$, and

$$
\begin{aligned}
\left|v_{n}(t)\right| & \leq e^{-i n\left(C(t)-C\left(t_{0}\right)\right)}\left|v_{n}\left(t_{0}\right)\right|+\sup _{s \in J}\left|f_{n}(s)\right| \ell(J) \\
& \leq e^{-n \eta_{2}}\left|v_{n}\left(t_{0}\right)\right|+\sup _{s \in J}\left|f_{n}(s)\right| \ell(J) .
\end{aligned}
$$

The result follows since $e^{-n \eta_{j}}\left|v_{n}\left(t_{0}\right)\right|$ is exponentially decaying in $n, j=1,2$.

Remark 2.12. If $t_{1}$ is an interior point of $J$ and $f_{n}$ and $v_{n}$ satisfy the assumptions of (2) (respectively, (3)) in the previous lemma, then $v_{n}(t)$ is exponentially decaying in $n$ for a slightly larger interval $\left[s_{1}, t_{2}\right] \cap J$ (respectively, the larger set $\left[s_{1}, t_{2}\right] \cap$ $\left.J \backslash\left(t_{0}-\varepsilon, t_{0}+\varepsilon\right)\right)$ having $t_{1}$ in its interior. Indeed, there exists $\varepsilon_{1}>0$ such that $e^{n \varepsilon_{1}} f_{n}(t)$ is still exponentially decaying in $n$ and uniformly in $t$. Thus, if $s_{1}<t_{1}$ is such that $B(t)-B(s) \leq \varepsilon_{1}$ for all $s, t \in\left[s_{1}, t_{1}\right] \cap J$ we get

$$
\begin{gathered}
\left|\int_{t_{0}}^{t} e^{-i n(C(t)-C(s))} f_{n}(s) d s\right| \leq\left|\int_{t_{0}}^{t} e^{n\left(B(t)-B(s)-\varepsilon_{1}\right)} e^{n \varepsilon_{1}}\right| f_{n}(s)|d s| \\
\leq e^{n \varepsilon_{1}} \sup _{s \in J}\left|f_{n}(s)\right| \ell(J) .
\end{gathered}
$$

A similar result holds for $t_{2}$. 
Remark 2.13. Results similar to the ones in Lemma 2.11 and in Remark 2.12 hold when $n \leq 0$ and $t_{1}$ and $t_{2}$ are defined instead by

$$
t_{1}=\inf \left\{t \in J ; B \text { is nonincreasing on }\left[t, t_{0}\right]\right\}
$$

and

$$
t_{2}=\sup \left\{t \in J ; B \text { is nondecreasing on }\left[t_{0}, t\right]\right\} .
$$

We now proceed to prove Lemma 2.1

By reordering we may assume that the singularity is to be put at $t_{0}$ and by translating we may assume that $t_{0}=0$. We may also assume that $A(0)=B(0)=0$.

The proof is divided into the cases $b_{0} \neq 0$ and $b_{0}=0$.

2.1. Case $b_{0} \neq 0$. We will prove only the case when $b_{0}<0$, the case $b_{0}>0$ being similar (one uses different, albeit similar, formulas for the solutions).

We will first give the proof in the case $r \geq 1$. The case $r=0$ is simpler, and at the end we will explain how its proof can be read from the proof of the case $r \geq 1$.

If we are to have $u \in \mathcal{D}^{\prime}\left(\mathbb{T}^{2}\right)$ and $L u=f \in C^{\omega}\left(\mathbb{T}^{2}\right)$, then we must have

$$
\partial_{t} \widehat{u}(t, n)+i n c(t) \widehat{u}(t, n)=\widehat{f}(t, n), \quad n \geq 1,
$$

and $\widehat{u}(t, n)$ must be given in terms of $\widehat{f}(t, n)$ by

or

$$
\begin{aligned}
\widehat{u}(t, n)=e^{-i n C(t)}\left[\int_{0}^{t} e^{i n C(s)} \widehat{f}(s, n) d s\right. & \\
& \left.+\left(e^{2 \pi i n c_{0}}-1\right)^{-1} \int_{0}^{2 \pi} e^{i n C(s)} \widehat{f}(s, n) d s\right]
\end{aligned}
$$

$$
\begin{aligned}
\widehat{u}(t, n) & =e^{-i n C(t)}\left(1-e^{-2 \pi i n c_{0}}\right)^{-1} \int_{0}^{t} e^{i n C(s)} \widehat{f}(s, n) d s \\
& +e^{-i n C(t)}\left(e^{i 2 \pi n c_{0}}-1\right)^{-1} \int_{t}^{2 \pi} e^{i n C(s)} \widehat{f}(s, n) d s .
\end{aligned}
$$

We also have a formula for expressing $\widehat{u}(t, n)$ in terms of $\widehat{u}\left(t^{\prime}, n\right)$, where $t, t^{\prime} \in \mathbb{R}$, namely,

$$
\widehat{u}(t, n)=e^{-i n\left\{C(t)-C\left(t^{\prime}\right)\right\}} \widehat{u}\left(t^{\prime}, n\right)+\int_{t^{\prime}}^{t} e^{-i n\{C(t)-C(s)\}} \widehat{f}(s, n) d s .
$$

Ideally we would like to construct $\widehat{u}(t, n)$, tempered in $n$ uniformly in $t$ so that we have

$$
\widehat{u}\left(t_{0}, n\right)=1, \quad \widehat{u}\left(t_{1}, n\right)=\cdots=\widehat{u}\left(t_{r}, n\right)=0 .
$$

Now by using (2.6), (2.3) and (2.5), we are led to find $u$ satisfying the following conditions:

$$
\begin{gathered}
\int_{0}^{t_{1}} e^{i n C(s)} \widehat{f}(s, n) d s=-1 ; \\
\int_{t_{k-1}}^{t_{k}} e^{i n C(s)} \widehat{f}(s, n) d s=0, \quad 2 \leq k \leq r \\
\int_{t_{r}}^{2 \pi} e^{i n[C(s)-C(2 \pi)]} \widehat{f}(s, n) d s=1 .
\end{gathered}
$$


We begin by defining $v_{n}(t)$ as the right-hand side of (2.3) (or (2.4)) with $\widehat{f}(\cdot, n)$ replaced by a piecewise continuous function $g_{n}$ given by

$$
g_{n}(t)=e^{i n(\psi(t)+i \varphi(t))}\left[C_{n} \chi_{\left[0, t_{1}\right)}+D_{n} \chi_{\left[t_{r}, 2 \pi\right)}\right],
$$

where

$$
\begin{gathered}
\varphi(t)=K\left(1-\cos \left(t-s_{1}\right)\right)\left(1-\cos \left(t-s_{r+1}\right)\right), \\
\psi(t)=a_{0} t-A(t)+\frac{a_{0}}{2} \sin \left(2 t-s_{1}-s_{r+1}\right), \quad \text { if } s_{r+1}-s_{1}=\pi
\end{gathered}
$$

and

$$
\psi(t)=a_{0} t-A(t)-\frac{a_{0}}{\cos \left(\left(s_{r+1}-s_{1}\right) / 2\right)} \sin \left(t-\frac{s_{1}+s_{r+1}}{2}\right), \quad \text { if }
$$

$s_{r+1}-s_{1} \neq \pi$, and, moreover, $K, C_{n}$, and $D_{n}$ will be chosen later.

Note that $A(t)-a_{0} t$ defines an element of $C^{\omega}\left(\mathbb{T}^{1}, \mathbb{R}\right)$.

By our choice of $g_{n}$ we have

$$
\int_{t_{k-1}}^{t_{k}} e^{i n C(s)} g_{n}(s) d s=0, \quad 2 \leq k \leq r .
$$

We now define $C_{n}$ and $D_{n}$ by the following equations:

$$
C_{n} \int_{0}^{t_{1}} e^{i n(C(s)+\psi(s)+i \varphi(s))} d s=-1
$$

and

$$
D_{n} \int_{t_{r}}^{2 \pi} e^{i n(C(s)+\psi(s)+i \varphi(s)-C(2 \pi))} d s=1 .
$$

We claim that both $C_{n}$ and $D_{n}$ are exponentially decaying as $n \rightarrow \infty$, uniformly in $t \in \mathbb{T}^{1}$. We prove this for $C_{n}$ employing the stationary phase method, as in $[\mathrm{Sj}]$.

Let

$$
\begin{gathered}
\beta(s)=-i(A(s)+i B(s)+\psi(s))+\varphi(s)-B\left(s_{1}\right)+i\left(A\left(s_{1}\right)+\psi\left(s_{1}\right)\right) \\
=B(s)+\varphi(s)-i(A(s)+\psi(s))-B\left(s_{1}\right)+i\left(A\left(s_{1}\right)+\psi\left(s_{1}\right)\right) .
\end{gathered}
$$

Hence, $\beta\left(s_{1}\right)=0$.

The function $\beta$ has a holomorphic extension to a complex neighborhood $V_{\delta}=$ $\left\{z=x+i y ;\left|x-s_{1}\right|<\delta,|y|<\delta\right\}$ of $s_{1}$, given by

$$
\tilde{\beta}(z)=B(z)+\varphi(z)-i(A(z)+\psi(z))-B\left(s_{1}\right)+i\left(A\left(s_{1}\right)+\psi\left(s_{1}\right)\right) .
$$

We have

$$
\tilde{\beta}^{\prime}\left(s_{1}\right)=b\left(s_{1}\right)+\varphi^{\prime}\left(s_{1}\right)-i\left(a\left(s_{1}\right)+\psi^{\prime}\left(s_{1}\right)\right)=0
$$

and

$$
\begin{gathered}
\tilde{\beta}^{\prime \prime}\left(s_{1}\right)=b^{\prime}\left(s_{1}\right)+\varphi^{\prime \prime}\left(s_{1}\right)-i\left(a^{\prime}\left(s_{1}\right)+\psi^{\prime \prime}\left(s_{1}\right)\right) \\
=b^{\prime}\left(s_{1}\right)+K\left(1-\cos \left(s_{1}-s_{r+1}\right)\right)-i\left(a^{\prime}\left(s_{1}\right)+\psi^{\prime \prime}\left(s_{1}\right)\right) .
\end{gathered}
$$

By taking $\delta>0$ small enough, we may suppose that $s_{1}$ is the only critical point of $\tilde{\beta}$ in $V_{\delta}$, and, for sufficiently large $K>0$, we have $\tilde{\beta}^{\prime \prime}\left(s_{1}\right) \neq 0$.

If $t \in I_{\delta}=\left[s_{1}-\delta, s_{1}+\delta\right]=\overline{V_{\delta}} \cap \mathbb{R}$, then

$$
\Re \tilde{\beta}(t)=B(t)-B\left(s_{1}\right)+\varphi(t) \geq B(t)-B\left(s_{1}\right) \geq 0
$$

if we take a smaller $\delta$, if necessary, since then $s_{1}$ is the unique point of global minimum of $B(t)-B\left(s_{1}\right), t \in I_{\delta}$. Finally, taking $0<\delta<\left(s_{r+1}-s_{1}\right) / 2$ we have $\varphi(t)>0$ if $t \in I_{\delta}, t \neq s_{1}, \varphi\left(s_{1}\right)=0$, and the same is true for $\Re \tilde{\beta}$. 
We may now apply the stationary phase theorem (see $[\mathrm{Sj}]$ ) to get that

$$
\int_{I_{\delta}} e^{-n \beta(s)} d s=\sqrt{2 \pi}\left(\tilde{\beta}^{\prime \prime}(0)\right)^{-1 / 2} n^{-1 / 2}\left(1+\mathcal{O}\left(n^{-1}\right)\right) .
$$

Let

$$
\eta=K(1-\cos \delta) \min \left\{1-\cos \left(s_{1}-s_{r+1}-\delta\right), 1-\cos \left(s_{1}-s_{r+1}+\delta\right)\right\} .
$$

We have $\eta>0$ and if $t \in\left[0, t_{1}\right] \backslash I_{\delta}$, then

$$
\Re \tilde{\beta}(t) \geq \varphi(t) \geq \eta \text {. }
$$

Thus,

Hence,

$$
\left|\int_{\left[0, t_{1}\right] \backslash I_{\delta}} e^{-n \beta(s)} d s\right| \leq 2 \pi e^{-n \eta} .
$$

$$
\begin{gathered}
\int_{0}^{t_{1}} e^{i n(C(s)+\psi(s)+i \varphi(s))} d s \\
=\sqrt{2 \pi} e^{-n\left(B\left(s_{1}\right)-i\left(A\left(s_{1}\right)+\psi\left(s_{1}\right)\right)\right)}\left(\tilde{\beta}^{\prime \prime}(0)\right)^{-1 / 2} n^{-1 / 2}\left(1+\mathcal{O}\left(n^{-1}\right)\right)
\end{gathered}
$$

which grows exponentially with $n \geq 1$ since $B\left(s_{1}\right)<0$.

Let

$$
h_{1, n}(t)=C_{n} \chi_{1}(t) e^{i n(\psi(t)+i \varphi(t))},
$$

where $\chi_{1}$ is a $C^{\infty} 2 \pi$-periodic function satisfying

(1) $0 \leq \chi_{1} \leq 1$

(2) $\chi_{1}(t)=0$ for $t_{1} \leq t \leq 2 \pi$,

(3) $\chi_{1}(t)=1$ for $t \in J_{1} \doteq\left[s_{1}-\delta_{1}, s_{1}+\delta_{1}\right] \subset I_{\delta}$.

Clearly,

$$
\int_{t_{k-1}}^{t_{k}} e^{i n C(s)} h_{1, n}(s) d s=\int_{t_{r}}^{2 \pi} e^{i n(C(s)-C(2 \pi))} h_{1, n}(s) d s=0, \quad 2 \leq k \leq r .
$$

By (2.11) we have

$$
\int_{0}^{t_{1}} e^{i n C(s)} h_{1, n}(s) d s=\alpha_{n}-1
$$

with

$$
\begin{gathered}
\alpha_{n} \doteq \int_{0}^{t_{1}} e^{i n C(s)}\left(h_{1, n}(s)-g_{n}(s)\right) d s \\
=C_{n} \int_{0}^{t_{1}} e^{i n(C(s)+\psi(s)+i \varphi(s))}\left(\chi_{1}(s)-1\right) d s .
\end{gathered}
$$

Thus

$$
\begin{gathered}
\left|\int_{0}^{t_{1}} e^{i n(C(s)+\psi(s)+i \varphi(s))}\left(\chi_{1}(s)-1\right) d s\right| \\
=\left|\int_{\left[0, t_{1}\right] \backslash J_{1}} e^{i n(C(s)+\psi(s)+i \varphi(s))}\left(\chi_{1}(s)-1\right) d s\right| \\
\quad \leq \int_{\left[0, t_{1}\right] \backslash I_{\delta}} e^{-n \Re \beta(s)} d s \leq 2 \pi e^{-n \eta},
\end{gathered}
$$

and, therefore, $\alpha_{n}$ decays exponentially. 
Setting $\lambda_{n} \doteq 1-\alpha_{n}$, we see that $\left|\lambda_{n}\right|$ is bounded above and below by positive constants and (2.12) can be rewritten as

$$
\int_{0}^{t_{1}} e^{i n C(s)} h_{1, n}(s) d s=-\lambda_{n} .
$$

Defining

$$
h_{r, n}(t)=D_{n} \chi_{r}(t) e^{i n(\psi(t)+i \varphi(t))},
$$

where $\chi_{r}$ is a $C^{\infty} 2 \pi$-periodic function satisfying

(1) $0 \leq \chi_{r} \leq 1$,

(2) $\chi_{r}(t)=0$ for $0 \leq t \leq t_{r}$,

(3) $\chi_{r}(t)=1$ for $t$ near $s_{r}$,

we have

$$
\int_{t_{k-1}}^{t_{k}} e^{i n C(s)} h_{r, n}(s) d s=0, \quad 1 \leq k \leq r
$$

In the same fashion as above one can prove that

$$
\mu_{n} \doteq \int_{t_{r}}^{2 \pi} e^{i n(C(s)-C(2 \pi))} h_{r, n}(s) d s
$$

has its absolute value bounded above and below by positive constants.

Setting $h_{n}=h_{1, n} / \lambda_{n}+h_{r, n} / \mu_{n}$, we obtain that equations (2.7), (2.8), and (2.9) are satisfied with $\widehat{f}(\cdot, n)$ replaced by $h_{n}$. Furthermore,

$$
\begin{aligned}
\left|h_{n}\right| \leq C\left(\left|h_{1, n}\right|+\left|h_{2, n}\right|\right) & \leq C\left(\left|e^{i n(\psi+i \varphi)} C_{n} \chi_{1}\right|+\left|e^{i n(\psi+i \varphi)} D_{n} \chi_{r}\right|\right) \\
& \leq C e^{-n \varphi}\left(\left|C_{n}\right|+\left|D_{n}\right|\right) \leq C\left(\left|C_{n}\right|+\left|D_{n}\right|\right),
\end{aligned}
$$

hence, $h_{n}$ is uniformly exponentially decaying.

Let $m=\min _{[0,2 \pi]} B$ and $M=\max _{[0,2 \pi]} B$. Note that $M \geq 0$. Fix $\sigma>M-m$ and for each $n \in \mathbb{N}$ let $\tilde{f}_{n}$ be a partial sum of the Fourier series of $h_{n}$ satisfying

$$
\left\|\widetilde{f}_{n}-h_{n}\right\|_{C^{0}} \leq e^{-\sigma n}
$$

By (2.13) and our choice of $\widetilde{f}_{n}$, we see that $\tilde{f}_{n}$ is uniformly exponentially decaying as $n \rightarrow+\infty$.

Let $\widetilde{u}_{n}(t)$ represent the right-hand side of $(2.3)$ with $\widehat{f}(s, n)$ replaced by $\widetilde{f}_{n}(s)$. We have

$$
\begin{gathered}
\widetilde{u}_{n}\left(t_{0}\right)=\left(e^{2 \pi i n c_{0}}-1\right)^{-1} \int_{0}^{2 \pi} e^{i n C(s)} \widetilde{f}_{n}(s) d s \\
=1+\left(e^{2 \pi i n c_{0}}-1\right)^{-1} \int_{0}^{2 \pi} e^{i n C(s)}\left[\widetilde{f}_{n}(s)-h_{n}(s)\right] d s=1+\gamma_{n}, \quad \text { say. }
\end{gathered}
$$

Since $\sigma>-m, b_{0}<0$, and

$$
\begin{gathered}
\left|\gamma_{n}\right| \leq\left|e^{2 \pi i n c_{0}}-1\right|^{-1} \int_{0}^{2 \pi} e^{-n B(s)}\left|\widetilde{f}_{n}(s)-h_{n}(s)\right| d s \\
\leq \frac{1}{e^{-2 \pi n b_{0}}-1} \int_{0}^{2 \pi} e^{-n m} e^{-n \sigma} d s \leq \frac{2 \pi}{e^{-2 \pi b_{0}}-1} e^{-(m+\sigma) n},
\end{gathered}
$$

we see that $\gamma_{n}$ is exponentially decaying as $n \rightarrow+\infty$. 
For $k=1, \ldots, r$,

$$
\begin{aligned}
& \widetilde{u}_{n}\left(t_{k}\right)=e^{-i n C\left(t_{k}\right)}\left[\int_{0}^{t_{k}} e^{i n C(s)}\right. {\left[\widetilde{f}_{n}(s)-h_{n}(s)\right] d s } \\
&\left.+\left(e^{i 2 \pi n c_{0}}-1\right)^{-1} \int_{0}^{2 \pi} e^{i n C(s)}\left[\widetilde{f}_{n}(s)-h_{n}(s)\right] d s\right]
\end{aligned}
$$

satisfies, as one can easily check,

$$
\left|\widetilde{u}_{n}\left(t_{k}\right)\right| \leq 2 \pi \frac{1}{1-e^{2 \pi b_{0}}} e^{(M-m-\sigma) n},
$$

which is also exponentially decaying as $n \rightarrow+\infty$.

Let $f_{n} \doteq \widetilde{f_{n}} /\left(1+\gamma_{n}\right)$. Since $f_{n}$ is uniformly exponentially decaying as $n \rightarrow+\infty$,

$$
f(t, x) \doteq \sum_{n \geq 1} f_{n}(t) e^{i n x}
$$

defines a real analytic function on $\mathbb{T}^{2}$ whose partial Fourier coefficients are given by $\widehat{f}(t, n)=f_{n}(t)$. Thus, if $u_{n}(t)$ stands for the right-hand side of (2.3), we get that $u_{n}\left(t_{0}\right)=1$ and $u_{n}\left(t_{k}\right)$ is exponentially decaying as $n \rightarrow+\infty$, for $k=1, \ldots, r$. Moreover, by Lemma 2.11,

$$
u(t, x) \doteq \sum_{n \geq 1} u_{n}(t) e^{i n x}
$$

defines an element of $\mathcal{D}^{\prime}\left(\mathbb{T}^{2}\right)$. A simple computation shows that the partial Fourier coefficients of $u$, which are given by $\widehat{u}(t, n)=u_{n}(t)$, satisfy (2.2), whence, $L u=f$.

Applying Lemma 2.11 one more time we see that the analytic singular support of $u$ is contained in $\left\{t_{0}\right\} \times \mathbb{T}^{1}$. In fact, sing $\operatorname{supp}_{\mathrm{A}}(u)=\left\{\left(t_{0}, 0\right)\right\}$ since $\widehat{u}\left(t_{0}, n\right)=1$, that is, $u\left(t_{0}, x\right)=\delta_{+}(x)$. This concludes the proof of the case $r \geq 1$.

We now now say a few words about the case $r=0$. All we need is $\widehat{u}(0, n)=1$ and we are led to

$$
\int_{0}^{2 \pi} e^{i n C(s)} \widehat{f}(s, n) d s=-1 .
$$

Instead of (2.10), take

$$
g_{n}(t)=C_{n} e^{i n(\psi(t)+i \varphi(t))}
$$

where now

$$
\begin{gathered}
\varphi(t)=K\left(1-\cos \left(t-s_{1}\right)\right), \\
\psi(t)=a_{0} t-A(t)-a_{0} \sin \left(t-s_{1}\right),
\end{gathered}
$$

and $K$ and $C_{n}$ are similarly chosen as in the case $r \geq 1$. We emphasize that in this case there is no need for approximations, that is, we can take $\widehat{f}(t, n)=g_{n}(t)=$ $h_{n}(t)$.

2.2. Case $b_{0}=0$. We will only treat the case $r \geq 1$. We divide the proof into the following three cases:

Case 1: $a_{0} \in \mathbb{R} \backslash \mathbb{Q}$. This case is quite similar to the case $b_{0} \neq 0$, since

$$
d_{n} \doteq e^{2 \pi i n c_{0}}-1=e^{2 \pi i n a_{0}}-1 \neq 0 \text {. }
$$

Note that $\left|d_{n}\right| \leq 2$. 
The only difference here is that we choose $\widetilde{f}_{n}$ satisfying

$$
\left\|\widetilde{f}_{n}-h_{n}\right\|_{C^{0}} \leq e^{-\sigma n}\left|d_{n}\right|
$$

instead of (2.14), to get

$$
\begin{gathered}
\left|\gamma_{n}\right| \leq\left|e^{2 \pi i n c_{0}}-1\right|^{-1} \int_{0}^{2 \pi} e^{-n B(s)}\left|\widetilde{f}_{n}(s)-h_{n}(s)\right| d s \\
\leq \int_{0}^{2 \pi} e^{-n m} e^{-n \sigma} d s \leq 2 \pi e^{-(m+\sigma) n} .
\end{gathered}
$$

The rest of the proof follows as in the case $b_{0} \neq 0$.

Case 2: $a_{0} \in \mathbb{Z}$. In this case we deal with

$$
\widehat{u}(t, n)=\int_{0}^{t} e^{i n[C(s)-C(t)]} \widehat{f}(s, n) d s+e^{-i n C(t)}
$$

instead of (2.3). Note that $e^{ \pm i n C(t)}$ is an element of $C^{\omega}(\mathbb{T})$.

We proceed as in the case $b_{0} \neq 0$ to get $\widetilde{f}_{n}$ as in (2.14).

Let $u_{n}(t)$ be the right-hand side of (2.15) with $f_{n} \doteq \widetilde{f}_{n}$ instead of $\widehat{f}(\cdot, n)$.

We have $u_{n}(0)=1$ and for $k=1, \ldots, r$,

$$
\begin{gathered}
u_{n}\left(t_{k}\right)=e^{-i n C\left(t_{k}\right)}\left[\int_{0}^{t_{k}} e^{i n C(s)} f_{n}(s) d s+1\right] \\
=e^{-i n C\left(t_{k}\right)} \int_{0}^{t_{k}} e^{i n C(s)}\left[f_{n}(s)-h_{n}(s)\right] d s,
\end{gathered}
$$

which leads to $\left|u_{n}\left(t_{k}\right)\right| \leq 2 \pi e^{n(M-m-\sigma)}$.

The rest of the proof follows as in previous cases.

Case 3: $a_{0} \in \mathbb{Q} \backslash \mathbb{Z}$. Write $a_{0}=p / q$ where $p \in \mathbb{Z}, q \in \mathbb{N}$, and g.c.d. $\{p, q\}=1$. Let $\mathcal{A} \doteq\{n q ; n \in \mathbb{N}\}$ and $\mathcal{B} \doteq \mathbb{N} \backslash \mathcal{A}$.

Let $f_{n}, u_{n}$ be as in the previous case with $a_{0}=q$. Define

$$
\widetilde{f}_{n}=\left\{\begin{array}{l}
f_{n} \text { if } n \in \mathcal{A}, \\
0 \text { if } n \in \mathcal{B},
\end{array} \quad \widetilde{u}_{n}= \begin{cases}u_{n} & \text { if } n \in \mathcal{A}, \\
0 & \text { if } n \in \mathcal{B} .\end{cases}\right.
$$

Then $\left(\partial_{t}+i n C^{\prime}\right) \widetilde{u}_{n}=\widetilde{f}_{n}$ and

$$
U_{0}(t, x) \doteq \sum_{n \in \mathbb{N}} \widetilde{u}_{n}(t) e^{i n x}=\sum_{n \in \mathcal{A}} u_{n}(t) e^{i n x}=\sum_{n \in \mathbb{N}} u_{n q}(t) e^{i n q x}
$$

defines an element of $\mathcal{D}^{\prime}\left(\mathbb{T}^{2}\right)$ with $L U_{0} \in C^{\omega}\left(\mathbb{T}^{2}\right)$ and sing $\operatorname{supp}_{A}\left(U_{0}\right)=\left\{\left(t_{0}, 0\right)\right\}$.

We note that if $n \in \mathcal{B}$, then $e^{2 \pi i n c_{0}}=e^{2 \pi i n p / q} \neq 1$. Thus, we are allowed to proceed as in the case $b_{0} \neq 0$ but working only with frequencies in $\mathcal{B}$. In this manner we obtain $U_{1} \in \mathcal{D}^{\prime}\left(\mathbb{T}^{2}\right)$ satisfying $\widehat{U}_{1}(t, n)=1$ if $n \in \mathcal{B}$ and $\widehat{U}_{1}(t, n)=0$ if $n \in \mathcal{A}$. Also, $L U_{1} \in C^{\omega}\left(\mathbb{T}^{2}\right)$, sing $\operatorname{supp}_{\mathrm{A}}\left(U_{1}\right)=\left\{\left(t_{0}, 0\right)\right\}$.

Finally, taking $U=U_{0}+U_{1}$ we get a singular solution of $L$ such that $U(0, x)=$ $\delta_{+}(x)$.

This completes the proof of Lemma 2.1. 


\section{Construction of singular solutions in the Case $b \equiv 0$}

In this section $L$ is a nonsingular, real vector field. Hence, if $L u=0$ and sing $\operatorname{supp}_{\mathrm{A}}(u)$ contains a point $p$, then it contains the complete integral curve of $L$ through $p$.

By using, if necessary, the change of variables $(t, x) \mapsto\left(t, x-A(t)+a_{0} t\right)$, we may assume $a(t)=a_{0}$. Thus, the study of $L u=f$ amounts to study the algebraic equation

$$
i\left(m+a_{0} n\right) \widehat{u}=\widehat{f}, \quad(m, n) \in \mathbb{Z}^{2} .
$$

We need a definition. We say that an irrational number $\alpha$ is exponential Liouville if there exist $\varepsilon>0$ and a sequence $\left\{\left(m_{k}, n_{k}\right)\right\}_{k>1}$ of points in $\mathbb{Z} \times \mathbb{N}$ satisfying $\left|\alpha+m_{k} / n_{k}\right| \leq e^{-\varepsilon n_{k}}$; the set of all such numbers is denoted by $\mathcal{E} \mathcal{L}$.

We divide the proof into the following three cases:

Case 1: $a_{0} \in \mathbb{Q}$. Write $a_{0}=p / q, q \in \mathbb{N}, p \in \mathbb{Z}$ with g.c.d. $\{p, q\}=1$.

Let $\mathcal{A} \doteq\left\{(m, n) \in \mathbb{Z}^{2} ; m+a_{0} n=0\right\}=\left\{(m, n) \in \mathbb{Z}^{2} ; q m+p n=0\right\}$.

Let $v(m, n)$ be a tempered sequence of complex numbers satisfying, for some $\varepsilon>0$ and $C>0,|v(m, n)| \leq C e^{-\varepsilon(|m|+|n|)}$, for all $(m, n) \notin \mathcal{A}$, i.e., $|q m+p n| \geq 1$.

Let $u(x, t)=\sum_{m, n \in \mathbb{Z}} v(m, n) e^{i(m t+n x)}$. It is clear that $u$ defines a distribution in $\mathbb{T}^{2}$

Set $g(m, n)=i\left(m+a_{0} n\right) \widehat{u}(m, n)=i\left(m+a_{0} n\right) v(m, n)$. We see that $g(m, n)=0$ for $(m, n) \in \mathcal{A}$ and for $(m, n) \notin \mathcal{A}$,

$$
|g(m, n)|=\left|m+a_{0} n\right||v(m, n)| \leq C\left|m+a_{0} n\right| e^{-\varepsilon(|m|+|n|)} .
$$

Since there exists $C_{1}>0$ such that $\left|m+a_{0} n\right| \leq C_{1} e^{\frac{\varepsilon}{2}(|m|+|n|)}$, we obtain

$$
|g(m, n)| \leq C_{0} e^{-\frac{\varepsilon}{2}(|m|+|n|)}
$$

for all $(m, n) \in \mathbb{Z}^{2}$, where $C_{0}=C C_{1}$.

Thus, $f(x, t)=\sum_{m, n \in \mathbb{Z}} g(m, n) e^{i(m t+n x)}$ defines an element of $C^{\omega}\left(\mathbb{T}^{2}\right)$ and $L u=$ $f$. In particular, if $v(m, n)$ is not exponentially decaying, then $u$ is a singular solution of $L$.

We now explain the location of the singularities. Given a distribution $v=v(x)$ in $\mathbb{T}^{1}$ with $v\left(x-\frac{2 \pi}{q}\right)=v(x)$ there exists a distribution $u=u(x, t)$ in $\mathbb{T}^{2}$ such that $L u=0$ and $u(x, 0)=v(x)$. In fact, we may take $u(x, t)=v(q x-p t)$.

Alternatively, if $v(x)=\sum_{\ell \in \mathbb{Z}} \widehat{v}(\ell) e^{-i q x \ell}$, then

$$
u(x, t)=v\left(x-\frac{p}{q} t\right)=\sum_{\ell \in \mathbb{Z}} \widehat{v}(\ell) e^{-i(q x-p t) \ell}
$$

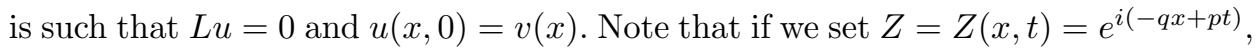
then $u=h \circ Z$ where

$$
h(\xi)=\sum_{\ell \in \mathbb{Z}} \widehat{v}(\ell) \xi^{\ell} .
$$

In any case, sing $\operatorname{supp}_{\mathrm{A}}(u)$ is equal to the union of all integral curves of $L$ which meet sing $\operatorname{supp}_{\mathrm{A}}(v)$.

Case 2: $a_{0} \in \mathbb{R} \backslash(\mathbb{Q} \cup \mathcal{E} \mathcal{L})$. In this case we prove that $L$ is GAH. Suppose $L u=f$ with $f \in C^{\omega}\left(\mathbb{T}^{2}\right)$ and $u \in \mathcal{D}^{\prime}\left(\mathbb{T}^{2}\right)$.

There exists $k_{0} \geq 0$ such that $|\widehat{f}(m, n)| \leq C_{0} e^{-\varepsilon(|m|+|n|)}$ for all integers $m, n$ with $|m|+|n| \geq k_{0}$. 
Since $a_{0} \notin \mathcal{E} \mathcal{L}$, there exists $k \geq k_{0}$ such that

$$
\left|a_{0}+\frac{m}{n}\right|>e^{-\frac{\varepsilon}{2}|n|}, \quad|m|+|n| \geq k .
$$

Hence, for all $(m, n)$ with $|m|+|n| \geq k$, we get

$$
\begin{aligned}
& \mid \widehat{u}(m, n \mid)=\frac{|\widehat{f}(m, n)|}{\left|m+a_{0} n\right|}=\frac{1}{|n|} \frac{|\widehat{f}(m, n)|}{\left|a_{0}+\frac{m}{n}\right|} \\
& \leq e^{\frac{\varepsilon}{2}|n|} C_{0} e^{-\varepsilon(|m|+|n|)}=C_{0} e^{-\frac{\varepsilon}{2}(|m|+|n|)} .
\end{aligned}
$$

Case 3: $a_{0} \in(\mathbb{R} \backslash \mathbb{Q}) \cap \mathcal{E} \mathcal{L}$. In this case there exist $\varepsilon>0$ and a sequence $\left\{\left(m_{k}, n_{k}\right)\right\}_{k \geq 1}$ of points in $\mathbb{Z} \times \mathbb{N}$ satisfying $\left|a_{0}+m_{k} / n_{k}\right| \leq e^{-\varepsilon n_{k}}$. Since $m_{k} / n_{k} \rightarrow$ $-a_{0}$, there exist $c>0$ and $k_{o}$ such that

$$
\frac{1}{c} n_{k} \leq\left|m_{k}\right| \leq c n_{k}, \quad \text { for all } k \geq k_{o} .
$$

Therefore, there exist positive constants $\varepsilon$ and $C$ such that $\left|a_{0} n_{k}+m_{k}\right| \leq$ $C e^{-\varepsilon\left(n_{k}+\left|m_{k}\right|\right)}$.

Let $\mathcal{A}=\left\{\left(m_{k}, n_{k}\right) ; k \geq 1\right\}$.

Let $v(m, n)$ be a tempered sequence of complex numbers satisfying, for some $\eta>0$ and $C_{0}>0,|v(m, n)| \leq C_{0} e^{-\eta(|m|+|n|)}$, for all $(m, n) \notin \mathcal{A}$.

Let $u(x, t)=\sum_{m, n \in \mathbb{Z}} v(m, n) e^{i(m t+n x)}$. It is clear that $u$ defines a distribution in $\mathbb{T}^{2}$.

Set $g(m, n)=i\left(m+a_{0} n\right) \widehat{u}(m, n)=i\left(m+a_{0} n\right) v(m, n)$.

Since $v(m, n)$ is tempered, there exists $C_{1}>0$ with $|v(m, n)| \leq C_{1} e^{\frac{\varepsilon}{2}(|m|+|n|)}$.

Thus, for $(m, n) \in \mathcal{A}$

$$
|g(m, n)| \leq C C_{1} e^{-\frac{\varepsilon}{2}(|m|+|n|)} .
$$

Now for $(m, n) \notin \mathcal{A}$,

$$
|g(m, n)|=\left|m+a_{0} n\right||v(m, n)| \leq C_{0}\left|m+a_{0} n\right| e^{-\eta(|m|+|n|)},
$$

and since there exists $C_{2}>0$ such that $\left|m+a_{0} n\right| \leq C_{2} e^{\frac{\eta}{2}(|m|+|n|)}$, we obtain

$$
|g(m, n)| \leq C_{0} C_{2} e^{-\frac{\eta}{2}(|m|+|n|)} .
$$

Thus, $f(x, t)=\sum_{m, n \in \mathbb{Z}} g(m, n) e^{i(m t+n x)}$ defines an element of $C^{\omega}\left(\mathbb{T}^{2}\right)$ and $L u=$ $f$. In particular, if $v(m, n)$ is not exponentially decaying, then $u$ is a singular solution of $L$; in fact, sing $\operatorname{supp}_{\mathrm{A}}(u)=\mathbb{T}^{2}$ since the orbit is dense.

\section{Appendix}

In this Appendix we present a one-dimensional periodic version of Theorem 8.4.14 in $[\mathrm{H}]$.

Lemma 4.1. Let $F$ be a nonempty closed subset of $\mathbb{T}^{1}$. Then there exists $v \in$ $\mathcal{D}^{\prime}\left(\mathbb{T}^{1}\right)$ such that $\mathrm{WF}_{\mathrm{A}}(v)=F \times \mathbb{R}_{+}$. Furthermore, $v$ can be chosen continuous and $v(x)=\sum_{n \geq 1} c_{n} e^{i n x}$.

Proof. Let $f(z)=z+(1-z) \log (1-z)$, where $\log$ is a branch of the logarithm defined in $\Omega=\mathbb{C} \backslash\{z=x+i y \in \mathbb{C} ; y=0, x \geq 1\}$. Note that $\lim _{z \in \Omega, z \rightarrow 1} f(z)=1$.

It is easy to see that

$$
f(z)=\sum_{n \geq 2} \frac{1}{n(n-1)} z^{n}, \quad \text { for }|z| \leq 1 .
$$


Let $M=\sum_{n \geq 2} 1 / n(n-1)$. Clearly, $|f(z)| \leq M$ in the closed unit disk $\bar{D}$.

Let $\left\{z_{k}\right\} \subset F$ be at most countable and dense in $F$ such that $z_{k} \neq z_{l}$ if $k \neq l$. Write $z_{k}=e^{i x_{k}}$ with $x_{k} \in[0,2 \pi)$.

Consider

$$
u(z)=\sum_{k \geq 1} \frac{1}{3^{k}} f\left(z e^{-i x_{k}}\right), \quad z \in \bar{D} .
$$

We see that $u$ is holomorphic in $D$ and continuous in $\bar{D}$. In particular, $v=b(u)$, the boundary value of $u$, defines an element of $\mathcal{D}^{\prime}\left(\mathbb{T}^{1}\right)$.

We have

$$
u^{\prime \prime}(z)=\sum_{k \geq 1} \frac{e^{-2 i x_{k}}}{3^{k}\left(1-z e^{-i x_{k}}\right)}, \quad \text { for } z \in D .
$$

For each $\ell \geq 1$ write

$$
u^{\prime \prime}(z)=\sum_{k=1}^{\ell-1} \frac{e^{-2 i x_{k}}}{3^{k}\left(1-z e^{-i x_{k}}\right)}+\frac{e^{-2 i x_{\ell}}}{3^{\ell}\left(1-z e^{-i x_{\ell}}\right)}+R(z),
$$

where

$$
R(z)=\sum_{k \geq \ell+1} \frac{e^{-2 i x_{k}}}{3^{k}\left(1-z e^{-i x_{k}}\right)}
$$

For $|z|<1$, we have

$$
|R(z)| \leq \frac{1}{1-|z|} \sum_{k \geq \ell+1} \frac{1}{3^{k}}=\frac{1}{2 \cdot 3^{\ell}} \cdot \frac{1}{1-|z|} .
$$

For $z=t e^{i x_{\ell}}, 0 \leq t<1$, we have

$$
\left|\frac{e^{-2 i x_{\ell}}}{3^{\ell}\left(1-z e^{-i x_{\ell}}\right)}\right|=\frac{1}{3^{\ell}} \frac{1}{1-t} .
$$

For such values of $z$ we obtain

$$
\left|\sum_{k \geq \ell} \frac{e^{-2 i x_{k}}}{3^{k}\left(1-z e^{-i x_{k}}\right)}\right| \geq \frac{1}{2 \cdot 3^{\ell}} \cdot \frac{1}{1-|z|},
$$

hence such a sum is unbounded when $z$ tends to $z_{\ell}$ along the ray $t z_{\ell}$.

Since

$$
\sum_{k=1}^{\ell-1} \frac{e^{-2 i x_{k}}}{3^{k}\left(1-z e^{-i x_{k}}\right)}
$$

is holomorphic, we conclude that $u$ cannot be extended holomorphically in a neighborhood of $z_{\ell}$. We have shown that $F \times \mathbb{R}_{+} \subset \mathrm{WF}_{\mathrm{A}}(v)$.

Let $\zeta=e^{i \xi} \in \mathbb{T}^{1} \backslash F$. Let $S=\left\{r e^{i x} ;|r-1|<\varepsilon,|x-\xi|<\varepsilon\right\}$ be such that $\bar{S} \cap F=\emptyset$, for some $\varepsilon>0$. Consider $D_{1}=\{z ;|z| \leq 1+\varepsilon\} \backslash e^{-i \xi} S$. Since $D_{1} \subset \Omega$ is compact we have $|f(z)| \leq C$ for some $C>0$ and all $z \in D_{1}$.

Since, for $z \in S$,

$$
\sum_{k \geq 1} \frac{1}{3^{k}}\left|f\left(z e^{-i x_{k}}\right)\right| \leq C / 2,
$$

it follows that $u$ can be continued holomorphically in a full neighborhood of $\zeta$. This means that $F \times \mathbb{R}_{+} \supset \mathrm{WF}_{\mathrm{A}}(v)$.

Remark 4.2. An analogous result is true for negative frequencies. 


\section{ACKNOWLEDGEMENT}

The authors are grateful to Jorge Hounie for his suggestion concerning the use, in the Appendix, of boundary values of holomorphic functions defined on the disk.

\section{REFERENCES}

[BT] Baouendi, M. S.; Trèves, F., A microlocal version of Bochner's tube theorem, Indiana Univ. Math. J. 31(6) (1982), 885-895. MR0674873 (84b:35025)

[B] Bergamasco, A., Remarks about global analytic hypoellipticity, Trans. Amer. Math. Soc. 351 (1999), 4113-4126. MR.1603878 (99m:35032)

[BCM] Bergamasco, A.; Cordaro, P.; Malagutti P., Globally hypoelliptic systems of vector fields, J. Funct. Anal. 114 (1993), 267-285. MR1223704 (94e:35048)

[BNZ1] Bergamasco, A.; Nunes, W.; Zani, S., Global analytic hypoellipticity and pseudoperiodic functions, Mat. Contemporanea 18 (2000), 43-57. MR1812862 (2001m:35062)

[BNZ2] Bergamasco, A.; Nunes, W.; Zani S., Global properties of a class of overdetermined systems, Journal of Functional Analysis 200 (2003), 31-64. MR1974087 (2004c:35295)

[DGY] Dickinson, D.; Gramchev, T.; Yoshino, M., Perturbations of vector fields on tori: resonant normal forms and Diophantine phenomena, Proc. Edinb. Math. Soc. 45 (2002), 731-759. MR.1933753 (2004h:37020)

[G] Greenfield, S., Hypoelliptic vector fields and continued fractions, Proc. Amer. Math. Soc. 31 (1972), 115-118. MR0301459(46:617)

[GW] Greenfield, S.; Wallach, N., Global hypoellipticity and Liouville numbers, Proc. Amer. Math. Soc. 31 (1972), 112-114. MR0296508 (45:5568)

[Hi] Himonas, A., Global analytic and Gevrey hypoellipticity of sublaplacians under Diophantine conditions, Proc. Amer. Math. Soc. 129 (2001), 2061-2067. MR1825918 (2002c:35074)

$[\mathrm{H}] \quad$ Hörmander, L., The analysis of linear partial differential operators. I. Distribution theory and Fourier analysis, Grundlehren der Mathematischen Wissenschaften, 256, SpringerVerlag, Berlin, 1983. MR0717035 (85g:35002a)

[Ho] Hounie, J., Globally hypoelliptic vector fields on compact surfaces, Comm. Partial Differential Equations 7 (1982), no. 4, 343-370. MR0652813 (83k:35025)

[M] Meziani, A., Hypoellipticity of nonsingular closed 1-forms on compact manifolds, Comm. Partial Differential Equations 27 (2002), no. 7-8, 1255-1269. MR1924466 (2003f:58002)

[Sj] Sjöstrand, J., Singularités analytiques microlocales, Astérisque, 95, 1-166, Soc. Math. France, Paris, 1982. MR0699623 (84m:58151)

[T1] Treves, F., Analytic hypoelliptic partial differential equations of principal type, Comm. Pure Appl. Math. 24 (1971), 537-570. MR0296509 (45:5569)

[T2] Treves, F., Hypoanalytic Structures, Princeton University Press, Princeton, NJ, 1992. MR.1200459(94e:35014)

Departamento de Matemática, Instituto de Ciências Matemáticas e de Computação - USP, Caixa Postal 668, São Carlos, SP, 13560-970 Brasil

E-mail address: apbergam@icmc.usp.br

Departamento de Matemática, Instituto de CiênCias Matemáticas e de Computação - USP, Caixa Postal 668, São Carlos, SP, 13560-970 Brasil

E-mail address: szani@icmc.usp.br 